\title{
The Impact of Inter-Site Distance and Time-to-Trigger on Handover Performance in LTE-A HetNets
}

\author{
Georgios Kollias*, Ferran Adelantado ${ }^{\dagger}$, Christos Verikoukis ${ }^{\ddagger}$ \\ * Iquadrat Informatica, Barcelona, Spain \\ $\dagger$ Universitat Oberta de Catalunya (UOC), Barcelona, Spain \\ $\ddagger$ Centre Tecnologic de Telecomunicacions de Catalunya (CTTC), Barcelona, Spain \\ Email: gkollias@iquadrat.com, ferranadelantado@uoc.edu, cveri@cttc.es
}

\begin{abstract}
Future cellular networks as envisaged by mobile operators, are expected to consist of macro cells overlaid with small nodes in dense architectures. These multi-tier deployments pose challenges to Mobility Management procedures like Handover, since traditional algorithms fail to keep up with heterogeneity, thus causing degradations in Handover performance. Finding ways to reduce unnecessary Handovers, mainly for fast moving users, and boost offloading of the lower speed ones to small cells, are subjects undergoing intense study. Most studies propose solutions based on the appropriate selection of the key parameters involved in the Handover procedure, such as the Hysteresis Margin and Time-to-Trigger. However, they do not take into account the impact that inter-site distance has on the Handover performance. In this framework, we study the dependency of the Handover procedure on the inter-site distance between a small cell and the overlaid macro cell in a two-tier deployment. Moreover, a Handover performance analysis in terms of Handover, Radio Link Failure, Handover Failure and Ping-Pong probabilities is carried out and evaluated through extensive simulations. Finally, the impact of TTT on the Handover performance is presented, while it is concluded that the appropriate TTT value should be selected according to inter-site distance, user profile (i.e speed) and overall mobility in the network automatically and in line with the concept of Self-Organized Networks (SON).
\end{abstract}

Index Terms-LTE-A, Mobility Management, Handover, Timeto-Trigger (TTT), Inter-Site Distance

\section{INTRODUCTION}

The need for mobile operators to support the demands of their subscribers due to capacity limitations of macro-only deployments, is considered the driving force for the introduction of small cells [1]. Hence, the transformation of cellular networks to dense multi-tier ones will be the next step in LTE A networks, the so-called Heterogeneous Networks (HetNets) [2]. The new architecture that will consist of macro cells, used for spatial coverage, and low power nodes (i.e. small cells) with the ability of offering high bitrates, is expected to improve the coverage, augment capacity and offer more flexibility to operators [3].

In this framework, study of handover (HO) has attracted large attention from both academia and industry [4]. It has been proven that using in HetNets the same set of HO parameters (i.e Hysteresis Margin $(H)$, Time-to-Trigger $(T T T)$ ) as in homogeneous networks degrades HO performance. Specifically, reduced coverage areas of small cells may result in frequent HOs, Ping-Pongs (PPs), Handover Failures (HOFs) and Radio Link Failures (RLFs), mainly for fast User Equipments (UEs) [5]. Therefore, the aforementioned transformation undergone in the networks' architecture imposes a reconsideration of the $\mathrm{HO}$ procedure to keep up with the increasing complexity. Given that, as stated above, the degradation of the HO metrics is tightly coupled with the UE profile (e.g. the UE speed), the proposed solutions must be UE specific and in alignment with the Self-Organized Networks (SON) concept.

The main objective of the studies in this area [6]-[14] is the reduction of the frequent (and unnecessary) HOs and the HOF probability, principally experienced by fast UEs. Specifically, they propose appropriate $H$ and TTT selection algorithms based on the UE profile (e.g speed, type of service etc.) and networks' conditions.

For instance, the work in [6] proposes an adaptive hysteresis algorithm that accounts for factors like UE speed, type of service and the load difference between the target and the serving cells, in order to reduce HOFs in a two-tier network. Similarly, an efficient handoff algorithm is presented in [7]. The idea is to combine both signals from the macro and the small cell, to compensate the uneven transmission power between nodes in different tiers, thereby creating an adaptive hysteresis margin and encouraging HOs to small cells.

A first attempt to shed some light on the relationship between HO performance and the appropriate TTT selection can be found in [8]. In it, the TTT is selected according to the UE speed and the target/source cells type to guarantee a minimum RLF probability, mainly caused by high TTT values.

Further studies on mobility performance in co-channel HetNet deployments are presented in [9] and [10]. Barbera et al. [9] investigate how TTT impacts on mobility performance. Furthermore a suitable adjustment of TTT according to UE speed and cell type is proposed in order to find a balance between RLF, HOF and PP probabilities. An extension of this work, which can be found in [10], focuses more on mobility. Through the proposal of enhanced mobility estimation schemes or UE autonomous HO decisions, their work 
maximizes the offloading of low speed UEs while, in parallel, prevents fast UEs from executing a $\mathrm{HO}$ to small cells.

Although the relationship between $\mathrm{HO}$ metrics is outlined in most of the aforementioned proposals, like in [11], the absence of solutions based on mathematical analysis is evident. Motivated by the above, the work in [12] uses a geometrical approach to derive closed-form expressions for HOF and PP probability as a function of UE speed, TTT and range expansion bias. The conclusion is that there is an optimal TTT value for a given UE and cell.

The geometric approach followed in [12] as well as the assumption regarding the circular area of the small cell [5], were used in [13] for expressing the HOF probability in a HetNet deployment as a function of the L3 sampling period. Finally, a work that investigates the dependency of the outbound $\mathrm{HOF}$ probability on parameters like UE speed, TTT and shadowed channel fading can be found in [14]. Closed-form expressions of the HOF probability as a function of the aforementioned parameters are derived by using stochastic geometry.

Despite the plethora of proposals on $\mathrm{HO}$ performance that stress out the significance of parameters such as type of cell, UE speed etc., the distance between source and target cell (i.e inter-site distance) has been overlooked.

The aim of this work is firstly to prove the dependency of the HO performance on the inter-site distance in a two-tier network. Secondly, to derive closed-form expressions for the different $\mathrm{HO}$ performance metrics as a function of inter-site distance and speed of UEs. Finally we aim to demonstrate how an appropriate TTT selection should be made, taking into account the inter-site distance and the UE's speed in order to offload traffic to small cells without degrading $\mathrm{HO}$ performance and ensuring that the RLF probability will not exceed $2 \%$ per $\mathrm{HO}$ as suggested in [8] and [15].

The rest of the paper is organized as follows: Section II gives insight on the problem under study while the HO performance analysis is carried out in Section III. Numerical results are presented in Section IV and Section V contains concluding remarks.

\section{PROBLEM Formulation}

HO performance is studied in a two tier deployment consisting of a small cell located at a distance $D$ (inter-site distance) from the center of the overlaid macro cell. UEs connected to the macro cell, cross the area of the small cell, moving on a straight line triggering a HO. This procedure is based on the A3 event, according to which a HO initiates when the received signal strength (RSS) from the target cell becomes an offset better than the source cell for a period equal to TTT [16]. The offset is known as Hysteresis Margin. The above definition can be expressed as follows

$$
R S S_{t} \geq R S S_{s}+H
$$

where $R S S_{t}$ and $R S S_{s}$ are the Received Signal Strength received from the target and the source cells, respectively, expressed in $\mathrm{dBm}$, while $H$ stands for the Hysteresis Margin, expressed in $\mathrm{dB}$. Note that the region where (1) holds, defines the coverage area of the small cell. As suggested in [5], [12] and [14], the region can be approximated as a circumference centered at the small cell site, depicted in Fig. 1 as the light shaded circle. Given this scenario, the $\mathrm{HO}$ performance is expressed in terms of HOFs, RLFs and PPs probabilities [5].

RLF is the loss of connection with the serving node as a result of degraded Signal to Interference Noise Ratio (SINR). Specifically, when SINR falls below a threshold, denoted as $Q_{\text {out }}$, and remains below $Q_{\text {in }}$ for 1 s, an RLF event is declared and the cell re-selection procedure is triggered [16], [17]. The values recommended by $3 \mathrm{GPP}$ for $Q_{\text {in }}$ and $Q_{\text {out }}$ are $-6 \mathrm{~dB}$ and $-8 \mathrm{~dB}$ respectively.

$\mathrm{HOF}$ is the interruption of the HO process due to degradation of the signal quality received from the serving node and is declared in three cases. Firstly, when the RLF timer, namely $\mathrm{T} 310$, is still running at the end of the HO preparation time $\left(T_{p}\right)$. Secondly, a HOF occurs if at the expiration of T310, TTT timer is active. Finally, if after HO execution time $\left(T_{e x}\right)$, target SINR is below $Q_{\text {out }}$, a HOF event is declared [5].

A successful HO results in a PP if the time a UE is connected to the target cell, namely Time of Stay (ToS), is less than Minimum-Time-of-Stay (MTS) before it handoffs back to the source cell. The MTS suggested by 3GPP is 1s [5].

Let us consider a generic UE located at the boundary of the small cell, defined in (1), at distance $d_{t}$ from the target cell, and $d_{s}$ from the source cell (both of them expressed in $\mathrm{km}$ ). The relationship between $d_{s}$ and $d_{t}$ is then described by

$$
d_{s}=\sqrt{D^{2}+2 D d_{t} \cos \left(\Theta_{\text {inner }}\right)+d_{t}^{2}}
$$

where $\Theta_{\text {inner }}$ is defined as the angle formed by the straight line joining the UE location and the small cell site, and the line joining the small cell and the macro cell sites (see Fig. $1)$. Focusing on (1), it can be reformulated as

$$
d_{t}^{\alpha_{t}} \leq d_{s}^{\alpha_{s}} \cdot 10^{\gamma-\frac{H}{10}}
$$

where $\alpha_{t}$ and $\alpha_{s}$ are the exponents of the corresponding path loss models and

$$
\gamma=\frac{\left(P_{T_{t}}-P_{T_{s}}\right)+\left(A_{s_{1}}-A_{t_{1}}\right)}{10}
$$

where $A_{s_{1}}$, and $A_{t_{1}}$ are distance independent components of the path loss models [18] [19], while $P_{T_{t}}$ and $P_{T_{s}}$ stand for the transmitted power from target and source cell respectively.

If we define the small cell radius $\left(R_{\Theta}\right)$ as the maximum $d_{t}$ (for a given $\Theta_{\text {inner }}$ ) for which (3) holds, the radius may be numerically calculated from the following expression

$$
R_{\Theta}=10^{\frac{\gamma-0.1 H}{\alpha_{t}}} \cdot\left(D^{2}+2 R_{\Theta} D \cos \left(\Theta_{\text {inner }}\right)+R_{\Theta}^{2}\right)^{\frac{\alpha_{s}}{2 \alpha_{t}}}
$$

At the edge of the region defined by (1), (3) or (4), TTT initiates and should expire within it in order for a HO to be executed successfully. However, if at expiration of TTT, the $\mathrm{UE}$ is located inside the small cell but the SINR received from the source cell is below $Q_{\text {out }}$, there is a HOF. This region, where $S I N R_{s} \leq Q_{\text {out }}$, is depicted in Fig. 1 as a white coloured circle (the inner circle, also known as HOF 
circle) and has a radius hereof denoted as $r_{\Theta}$. Similarly to (4), $r_{\Theta}$ is given by

$$
r_{\Theta}=10^{\frac{\gamma+0.1 Q_{\text {out }}}{\alpha_{t}}} \cdot\left(D^{2}+2 r_{\Theta} D \cos \left(\Theta_{\text {inner }}\right)+r_{\Theta}^{2}\right)^{\frac{\alpha_{s}}{2 \alpha_{t}}}
$$

Once a UE is associated to the small cell, the outbound $\mathrm{HO}$ procedure starts (i.e. the TTT countdown) when (1) holds. Note, however, that in this case the macro cell plays the role of target cell and the small cell is the source cell. Following the same reasoning, the radius of the outbound HO, namely $S_{\Theta}$, (the dark shaded circle in Fig. 1) may be calculated numerically from the following

$$
S_{\Theta}=10^{\frac{-\gamma+0.1 H^{\prime}}{\alpha_{s}}} \cdot\left(D^{2}+2 S_{\Theta} D \cos \left(\Theta_{\text {inner }}\right)+S_{\Theta}^{2}\right)^{\frac{\alpha_{t}}{2 \alpha_{s}}}
$$

where $H^{\prime}$ is the hysteresis margin used to handoff from the small cell to the macro cell. It is worth noting that all these radii, i.e. $R_{\Theta}, r_{\Theta}$ and $S_{\Theta}$, do depend on the inter-site distance $D$ and $\Theta_{\text {inner. }}$. As UEs are assumed to be distributed randomly around the scenario, the mean radius $R$ can be expressed as

$$
R=\mathbb{E}\left[R_{\Theta}\right]=\frac{1}{\pi} \int_{0}^{\pi} R_{\Theta} d \Theta_{\text {inner }}
$$

Likewise, the mean of $r_{\Theta}$ and $S_{\Theta}$ are given by $r=\mathbb{E}\left[r_{\Theta}\right]$ and $S=\mathbb{E}\left[S_{\Theta}\right]$, respectively. In Section III, the analytical expressions for inbound $\mathrm{HO}\left(P_{H O}\right), \operatorname{RLF}\left(P_{R L F}\right), \mathrm{HOF}\left(P_{H O F}\right)$ and PP $\left(P_{P P}\right)$ probabilities are derived as a function of $R, r$ and $S$ to further analyze the impact of UE's speed and $D$ on the appropriate selection of TTT values. Although there are other factors that could impact the performance of the described probabilities, e.g. the L3 filter or the measurement errors [13], the presented model will assume perfect measurement and no L3 delay to focus on the effect of the TTT and inter-site distance

\section{Analytical Model}

Let us focus on the same generic UE, located at distance $R$ from the center of the small cell. This UE, moving at speed $v$, crosses the small cell coverage area with an entry angle $\theta_{e}$ (see Fig. 1). Due to symmetry, the whole analysis will be done hereafter for $0 \leq \theta_{e} \leq \frac{\pi}{2}$. In this situation, this UE may perform a HO, suffer from a HOF, an RLF, or/and a PP. Before proceeding with the analysis of the aforementioned probabilities, it is necessary to define a set of important angles.

First, let $\left(T T T+T_{p}\right)$ be the time needed for an inbound $\mathrm{HO}$ to be completed, from now on denoted as $T$. The distance covered by the UE during time $T$ is then equal to $v T$. Accordingly, if we define $\theta_{i}$ as the maximum entry angle for which the inbound $\mathrm{HO}$ can be completed (i.e. the distance covered within the small cell coverage area is, at least, $v T$, as shown in Fig. 1), it may be expressed as

$$
\theta_{i}=\arccos \left(\frac{v T}{2 R}\right)
$$

with $0 \leq v \leq \frac{2 R}{T}$. If $v>\frac{2 R}{T}$, the UE will not handoff to the small cell regardless of the entry angle.

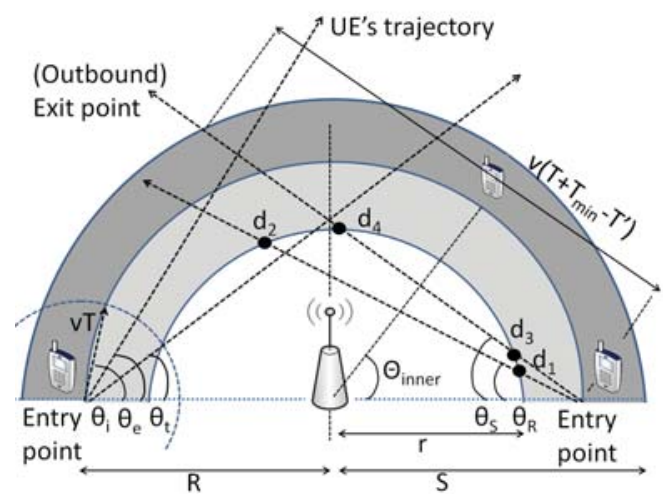

Fig. 1. Small cell area and considered angles

The second angle to consider, $\theta_{t}$, is defined as the maximum angle for which $T$ expires within the HOF circle (Fig. 1). By using the Cosine Law, $\theta_{t}$ may be expressed as

$$
\theta_{t}=\arccos \left(\frac{(v T)^{2}+R^{2}-r^{2}}{2 v T R}\right)
$$

where $\frac{R-r}{T} \leq v \leq \frac{R+r}{T}$. Note that, if $v<\frac{R-r}{T}$, the UE will not suffer from a HOF, even if the entry angle $\theta_{e}=0$. Likewise, if $v>\frac{R+r}{T}$, there will not be a HOF either.

As explained in Section II, the RLF occurs after the UE has moved over the HOF region for more than a particular time, usually $1 \mathrm{~s}$. If we define this maximum time as $T_{R}$, the UE will suffer from a RLF if the distance covered within the HOF circle is above $v T_{R}$ while a $\mathrm{HO}$ is not yet successfully completed. Thus, the maximum angle for which the distance covered by a UE within the HOF region is larger than $v T_{R}$, is given by

$$
\theta_{R}=\arcsin \left(\sqrt{\frac{r^{2}}{R^{2}}-\left(\frac{v T_{R}}{2 R}\right)^{2}}\right)
$$

where $\frac{R-r}{T} \leq v \leq \frac{2 r}{T_{R}}$. Otherwise $\theta_{R}$ is not defined and a UE will not suffer from RLF. It is worth noting that, for a given entry angle $\theta_{e}=\theta_{R}$, the UE intersects the HOF circle at two points. Thus, if we denote the distance covered by the UE from the entry point to the first intersection as $d_{1}$ and to the second intersection as $d_{2}$, they may be expressed as

$$
\begin{gathered}
d_{1}=\sqrt{R^{2}-r^{2}+\left(\frac{v T_{R}}{2}\right)^{2}}-\frac{v T_{R}}{2} \\
d_{2}=d_{1}+v T_{R}
\end{gathered}
$$

These two points will be used later on to characterize the HO, the RLF and the PP probabilities. As initially stated in Section II, a UE causes a PP if, after completing an inbound HO (which entire process lasts $T$ ), the ToS is below the MTS, hereafter referred to as $T_{\min }$. Note that the outbound $\mathrm{HO}$ process is triggered when the UE trajectory intersects the circumference with radius $S$, and it is finally completed after the outbound $\left(T T T+T_{p}\right)$ expires, namely $T^{\prime}$. Therefore, a 
UE will cause a PP if, after completing a successful HO, it covers a total distance within the small cell (from the entry point to the exit point) shorter than $v\left(T+T_{\min }-T^{\prime}\right)$. Hence, the maximum entry angle for which a UE will not cause a PP $\left(\theta_{s}\right)$ is given by

$$
\theta_{s}=\arccos \left(\frac{R^{2}-S^{2}+v^{2}\left(T+T_{m i n}-T^{\prime}\right)^{2}}{2 R v\left(T+T_{m i n}-T^{\prime}\right)}\right)
$$

where,

$$
\frac{\sqrt{S^{2}-R^{2}}}{T+T_{\text {min }}-T^{\prime}} \leq v \leq \frac{R+S}{T+T_{\text {min }}-T^{\prime}}
$$

It may be easily calculated that a UE trajectory with an entry angle $\theta_{e}=\theta_{s}$ will not intersect the HOF circle if

$$
v<\frac{\sqrt{R^{2}-r^{2}}+\sqrt{S^{2}-r^{2}}}{T+T_{\text {min }}-T^{\prime}}
$$

Then, if this UE trajectory does intersect the HOF circle, the distances covered from the entry point to the HOF circle intersections, denoted as $d_{3}$ and $d_{4}$, can be expressed as

$$
\begin{aligned}
& d_{3}=\delta-\sqrt{r^{2}-R^{2}+d^{2}} \\
& d_{4}=\delta+\sqrt{r^{2}-R^{2}+d^{2}}
\end{aligned}
$$

where

$$
\delta=\frac{R^{2}-S^{2}+v^{2}\left(T+T_{\min }-T^{\prime}\right)^{2}}{2 v\left(T+T_{\min }-T^{\prime}\right)}
$$

Based on the aforementioned definitions, expressions for $P_{H O}, P_{H O F}, P_{R L F}$ and $P_{P P}$ will be derived in the sequel.

\section{A. Inbound Handover Probability}

According to the definitions previously stated, a UE can only perform a successful $\mathrm{HO}$ if the entry angle is smaller than $\theta_{i}$. Otherwise, the UE's TTT timer expires after leaving the small cell coverage area and the $\mathrm{HO}$ process is not completed. However, and despite having $\theta_{e}<\theta_{i}$, the HO could not be completed successfully due to either a HOF (i.e. $\theta_{t} \geq \theta_{e}$ ) or an RLF (i.e. $\theta_{R} \geq \theta_{e}$ and $\frac{R-r}{T} \leq v$ ). If we define $\Theta_{H O}^{v}$ as the set of entry angles that, for a given $v$, result in a successful $\mathrm{HO}$, the $P_{H O}$ is then given by

$$
P_{H O}=\frac{2}{\pi} \int_{\theta_{e} \in \Theta_{H O}^{v}} \theta_{e} d \theta_{e}
$$

With regard to $\Theta_{H O}^{v}$, it will be $\Theta_{H O}^{v}=\left[0, \theta_{i}\right]$ when the $\mathrm{HO}$ is completed before reaching the HOF circle (i.e. $v<\frac{R-r}{T}$ ), $\Theta_{H O}^{v}=\left[\theta_{t}, \theta_{i}\right]$ when $T$ expires inside the HOF circle (i.e $\left.\frac{R-r}{T} \leq v \leq \frac{R+r}{T}\right)$ and $\theta_{R}$ does not exist, $\Theta_{H O}^{v}=\left[\theta_{t}, \theta_{i}\right]$ while $\theta_{R}<\theta_{t}$ (if $\theta_{R}$ exists), $\Theta_{H O}^{v}=\left[\theta_{R}, \theta_{i}\right]$ if $v T \geq d_{2}$ and $\theta_{R}$ exists, and finally $\Theta_{H O}^{v}=\left[0, \theta_{i}\right]$ when $\theta_{R}$ does not exist and $\frac{R+r}{T}<v \leq \frac{2 R}{T}$. Thus,

$$
P_{H O}= \begin{cases}\frac{2}{\pi} \theta_{i} & \text { if } 0 \leq v<\frac{R-r}{T} \\ \frac{2}{\pi}\left(\theta_{i}-\theta_{t}\right) & \text { if } \frac{R-r}{T} \leq v \leq \frac{R+r}{T} \text { and } \nexists \theta_{R} \\ \frac{2}{\pi}\left(\theta_{i}-\theta_{t}\right) & \text { if } \frac{R-r}{T} \leq v<\frac{d_{2}}{T} \text { and } \exists \theta_{R} \\ \frac{2}{\pi}\left(\theta_{i}-\theta_{R}\right) & \text { if } \frac{d_{2}}{T} \leq v \leq \frac{2 R}{T} \text { and } \exists \theta_{R} \\ \frac{2}{\pi} \theta_{i} & \text { if } \frac{R+r}{T}<v \leq \frac{2 R}{T} \text { and } \nexists \theta_{R} \\ 0 & \text { otherwise }\end{cases}
$$

\section{B. Handover Failure Probability}

A HOF occurs if $T$ expires within the HOF circle. Analogously to $P_{H O}$, the set of entry angles for which a UE suffers from a HOF, namely $\Theta_{H O F}^{v}$, is defined as $\Theta_{H O F}^{v}=\left[0, \theta_{t}\right]$ for $\frac{R-r}{T} \leq v \leq \frac{R+r}{T}$ and $\Theta_{H O F}^{v}=\emptyset$ otherwise. Therefore,

$$
P_{\text {HOF }}= \begin{cases}\frac{2}{\pi} \theta_{t} & \text { if } \frac{R-r}{T} \leq v \leq \frac{R+r}{T} \\ 0 & \text { otherwise }\end{cases}
$$

\section{Radio Link Failure Probability}

The probability of RLF $\left(P_{R L F}\right)$ is different from 0 only when $\theta_{R}$ exists. Thus, assuming that $\theta_{R}$ exists, the RLF presents two possible situations (see Fig. 1): first, if $R-r \leq$ $v T \leq d_{1}$, there is a RLF only after a previous HOF (and consequently, the set of angles that cause a RLF is $\Theta_{R L F}^{v}=\left[0, \theta_{t}\right]$ ); second, when $d_{1}<v T \leq 2 R$, only UEs with an entry angle below $\theta_{R}$ suffer from RLF (i.e. $\Theta_{R L F}^{v}=\left[0, \theta_{R}\right]$ ). Therefore,

$$
P_{R L F}= \begin{cases}\frac{2}{\pi} \theta_{t} & \text { if } \frac{R-r}{T}<v \leq \frac{d_{1}}{T} \\ \frac{2}{\pi} \theta_{R} & \text { if } \frac{d_{1}}{T}<v \leq \frac{2 R}{T} \\ 0 & \text { otherwise }\end{cases}
$$

\section{Ping Pong Probability}

The Ping Pong probability $\left(P_{P P}\right)$ is defined as the probability that, for a UE that has completed a successful handover to the small cell, the duration of the connection to the small cell is below the MTS, denoted as $T_{m i n}$. As the final expression of the $P_{P P}$ is complex, let us define a set of conditions based on the definitions stated at the beginning of Section III.

$$
\begin{array}{cc}
C_{1}: & \frac{R-r}{T} \leq v \leq \frac{2 r}{T_{R}} \\
C_{2}: & \frac{\sqrt{S^{2}-R^{2}}}{T+T_{m i n}-T^{\prime}} \leq v \leq \frac{R+S}{T+T_{m i n}-T^{\prime}} \\
C_{3}: & v<\frac{\sqrt{S^{2}-R^{2}}}{T+T_{\min }-T^{\prime}} \\
C_{4}: & v>\frac{R+S}{T+T_{\min }-T^{\prime}} \\
C_{5}: & v<\frac{\sqrt{R^{2}-r^{2}}+\sqrt{S^{2}-r^{2}}}{T+T_{m i n}-T^{\prime}} \\
C_{6}: & v \leq \sqrt{\frac{S^{2}-R^{2}}{\left(T+T_{m i n}-T^{\prime}\right)\left(T_{\min }-T^{\prime}\right)}} \\
C_{7}: & d_{2} \leq d_{4} \\
C_{8}: & \frac{d_{3}}{T}<v<\frac{d_{4}}{T}
\end{array}
$$

The first condition, $C_{1}$, defines the range of $v$ for which $\theta_{R}$ exists. Note that, if there is an RLF, there cannot be a PP. Condition $C_{2}$ guarantees the existence of $\theta_{s}$. If $C_{2}$ does not hold, either $C_{3}$ or $C_{4}$ must be true. In particular, $C_{3}$ means that the ToS of the UE in the small cell is long enough (due to small $v$ ) to achieve $P_{P P}=0$. As for $C_{4}$, the speed is too high and so $P_{P P}=1$. When $C_{5}$ is accomplished, the trajectory of a UE with $\theta_{e}=\theta_{s}$ does not intersect the HOF circle. Condition $C_{6}$ is particularly important, since it means that $\theta_{s} \geq \theta_{i}$. It is worth noting that, for all $\theta_{e} \leq \theta_{s}$, there is not any PP, whereas for all UEs with $\theta_{e}>\theta_{i}$ there cannot be a HO. Hence, if $C_{6}$ holds, the $P_{P P}=0$. Finally, $C_{7}$ is equivalent to $\theta_{R} \geq \theta_{s}$, whereas $C_{8}$ is equivalent to $\theta_{t}>\theta_{s}$.

In the following, we will denote the complement of a condition $C_{i}$ as $C_{i}^{\prime}$. For instance, when $C_{2}$ holds, neither $C_{3}$ 
nor $C_{4}$ do. Therefore, $C_{2}^{\prime}=C_{3} \cup C_{4}$. Based on the definitions, $P_{P P}$ may be expressed as

$$
P_{P P}= \begin{cases}0 & \text { if } C_{3} \\ 1 & \text { if } C_{4} \\ 0 & \text { if } C_{2} \cap C_{6} \\ \frac{\frac{2}{\pi}\left(\theta_{i}-\theta_{t}\right)}{\left.P_{H O}\right)} & \text { if } C_{1}^{\prime} \cap C_{2} \cap C_{5}^{\prime} \cap C_{6}^{\prime} \cap C_{8} \\ \frac{\frac{2}{\pi}\left(\theta_{i}-\theta_{t}\right)}{P_{H O}} & \text { if } C_{1} \cap C_{2} \cap C_{5}^{\prime} \cap C_{6}^{\prime} \cap C_{7}^{\prime} \cap C_{8} \\ 1 & \text { if } C_{1} \cap C_{2} \cap C_{5}^{\prime} \cap C_{6}^{\prime} \cap C_{7} \\ \frac{\frac{2}{\pi}\left(\theta_{i}-\theta_{s}\right)}{P_{H O}} & \text { otherwise }\end{cases}
$$

\section{NUMERICAL RESUlTS}

The scenario under study consists of a small cell overlaid with a macro cell, with a distance between the macro cell site and the small cell site $(D)$ that ranges from $40 \mathrm{~m}$ to $240 \mathrm{~m}$. UEs are spread randomly over the layout moving at $60 \mathrm{~km} / \mathrm{h}$ (a very high speed in an urban scenario) and heading for the small cell coverage area with a random entry angle and moving on a straight line. The transmitted power of the macro and the small cell is 46 and $20 \mathrm{dBm}$, respectively [18][19]. Although a range of 16 possible TTT values are defined in [16], only the results (both simulated and analytical) for an illustrative subset of them have been included (specifically, TTT equal to 128 , 256, 320 and $512 \mathrm{~ms}$ ). The rest of the simulation parameters can be found in Table I.

TABLE I

SIMULATION PARAMETERS

\begin{tabular}{|c|c|}
\hline Bandwidth & $10 \mathrm{MHz}$ \\
\hline Macro and Small Cell Frequency & $2 \mathrm{GHz}$ \\
\hline Macro cell Path-Loss & $128.1+37.6 \log _{10}(d \mathrm{~km})$ \\
\hline Small cell Path-Loss & $140.7+36.7 \log _{10}(\mathrm{~d} \mathrm{~km})$ \\
\hline Macro cell transmitted power & $26 \mathrm{dBm}$ \\
\hline Small cell transmitted power & $3 \mathrm{dBm}$ \\
\hline HO A3 Hysteresis Margin & $50 \mathrm{~dB}$ \\
\hline TTT values & $40 \mathrm{~ms}$ \\
\hline HO Preparation Time & $256,320,512 \mathrm{~ms}$ \\
\hline HO Execution Time &
\end{tabular}

Focusing on $P_{H O}$ and $P_{H O F}$, depicted in Fig. 2 and Fig. 3, it is important to point out their dependency on $\theta_{i}, \theta_{t}, R$ and $r$. In particular, the inspection of (20) reveals that $P_{H O}$ grows when $\theta_{i}$ grows and/or $\theta_{t}$ falls. Thus, for a given $v$ and $T$, $\theta_{i}$ rises when $R$ grows (or in other other words, when $D$ is increased) according to (8). Conversely, it may be observed in (9) that $\theta_{t}$ decreases as $R$ rises. These two factors result in the upward trend shown in Fig. 2.

As for HOF probability, $P_{H O F} \neq 0$ if $\frac{R-r}{T} \leq v \leq \frac{R+r}{T}$. Therefore, it is tightly coupled with the size of the coverage area $(R)$ and the HOF region $(r)$. The numerical solution of (7) shows that in the simulated scenario the ratio $\frac{r}{R}=\beta$ remains approximately constant and equal to 0.73 for the whole range of $D$. Based on this, and making use of (21), the $P_{H O F}$ will be different from 0 when

$$
\frac{v T}{1+\beta} \leq R \leq \frac{v T}{1-\beta}
$$

Fig. 3 displays the performance of $P_{H O F}$ described in (32). Specifically, $P_{H O F}$ presents an initial upward trend (commenced when $R=\frac{v T}{1+\beta}$ ) followed by a subsequent decreasing trend (that results in $P_{H O F}=0$ when $R>\frac{v T}{1-\beta}$ ). This also explains the peaks observed in Fig. 2 for $D \simeq 50 \mathrm{~m}$ when TTT equals $320 \mathrm{~ms}$, and $D \simeq 80 \mathrm{~m}$ when TTT is $512 \mathrm{~ms}$. Initially, the $P_{H O}$ for these TTT values has only the contribution of $\theta_{i}$, since $v T<R-r$ (see the expression in (20)), thereby resulting in an abrupt increase. However, when $R=\frac{v T}{1+\beta}$ the increase of $\theta_{i}$ is counteracted by $\theta_{t}$, causing the aforementioned peak.

Finally, Fig. 4 and Fig. 5 complement the analysis with the $P_{R L F}$ and the $P_{P P}$, respectively. As expected, it may be observed that $P_{R L F}=0$ as long as HOF region is not large enough to yield $v T_{R} \leq 2 r$ (the necessary condition stated in (10) to have RLFs). Moreover, $T$ must be long enough so that the UE does not complete successfully a HO, while the time spent inside the HOF region is at least equal to $T_{R}$. Therefore, $P_{R L F} \neq 0$ for large $D$ and $T$.

With regard to $P_{P P}$, it is correlated with $P_{H O}$, since there cannot be a PP if there is not any previous HO. Fig. 5 reveals that, for a given $v$, the performance in terms of PP depends on two key parameters: $D$ and $T$. In particular, when the small cell is deployed close to the macro cell site (small $D$ values) and there is a successful HO, both $R$ and $S$ are small and so the UE is prone to short ToS and the consequent PP. On the contrary, $P_{P P}$ falls for the same reason when the distance between the macro and the small cell rises. The impact of $T$ on $P_{P P}$ is in turn the opposite, since larger $T$ leads to longer HO delay, and therefore shorter ToS in the small cell (Fig. 5).

Fig. 2-Fig. 5 demonstrate that the appropriate selection of TTT, as a function of $v$ and $D$, presents important challenges to cope with the conflicting trends experienced by the different HO performance probabilities. Note that these challenges are not addressed by related works where TTT remains constant for different inter-site distances. In more detail, the TTT selection should start with the limitation of the maximum $P_{R L F}$ (limited to $2 \%$ according to [8]). Then, the final TTT value should be selected based on the maximum acceptable $P_{H O F}$ and $P_{P P}$. It is worth noting that there is a degree of freedom in the selection of the TTT. This degree of freedom allows the possibility to manage the usage of the small cell. Thus, in a scenario characterized by high mobility, an increase of $P_{H O}$ of UEs moving at high speed results in a higher small cell usage. Conversely, in low mobility scenarios, $P_{H O}$ for high $v$ should be decreased to reduce the small cell usage (in case of overload in the small cell).

\section{CONCLUSIONS}

In this work, a Handover performance analysis in terms of $P_{H O}, P_{H O F}, P_{R L F}$, and $P_{P P}$ was carried out. Specifically, we have proven the dependency of the Handover on the intersite distance between the small cell and the overlaid macro 


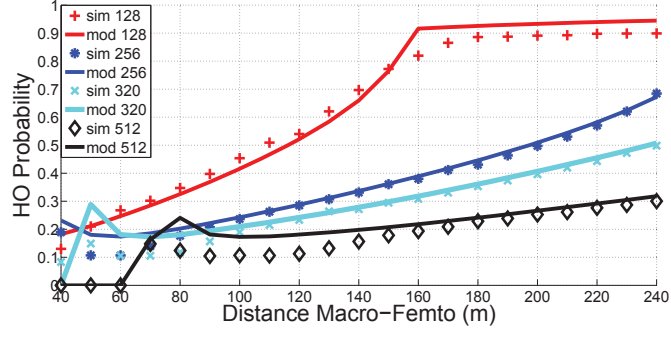

Fig. 2. HO probability $\left(P_{H O}\right)$

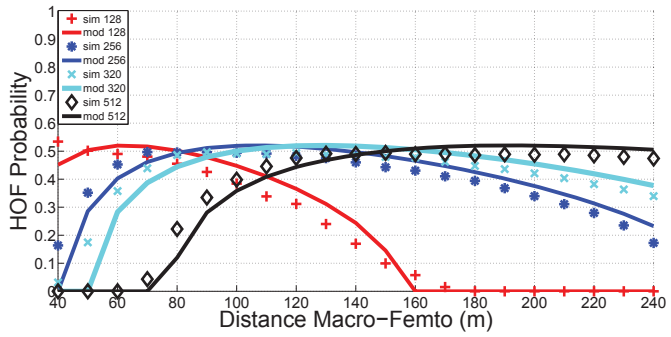

Fig. 3. HOF probability $\left(P_{H O F}\right)$

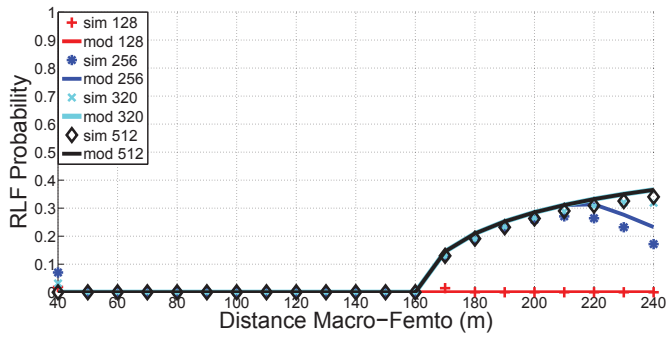

Fig. 4. RLF probability $\left(P_{R L F}\right)$

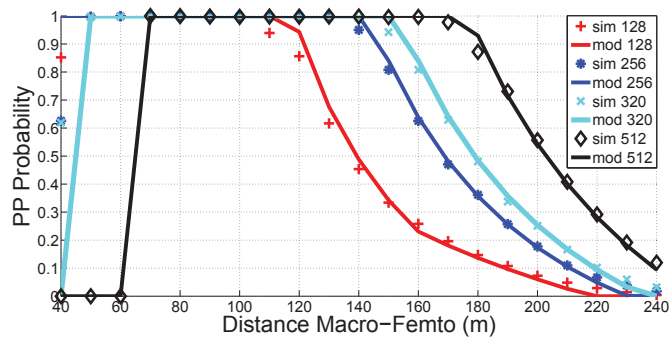

Fig. 5. PP probability $\left(P_{P P}\right)$

cell center in a two-tier scenario Furthermore, closed-form expressions for the aforementioned probabilities were derived as a function of inter-site distance and speed of the UEs. Finally, it has been shown that the appropriate TTT selection, based on inter-site distance and UE's profile, is essential for maintaining the conflicting trends of different $\mathrm{HO}$ performance probabilities. Therefore, it should be selected in a more flexible way, on a UE basis, and adjusted to network's characteristics and objectives to enhance $\mathrm{HO}$ performance.

\section{ACKNOWLEDGEMENT}

This work has been funded by the MITN Project CROSSFIRE (PITN-GA-2012-317126) and was supported in part by the Generalitat de Catalunya under grant 2014-SGR-1551

\section{REFERENCES}

[1] J. Hoadley, P. Maveddat, "Enabling small cell deployment with HetNet", Wireless Communications, IEEE, vol.19, no.2, pp.4,5, April 2012

[2] T. Nakamura, S. Nagata, A. Benjebbour, Y. Kishiyama, Tang Hai, Shen Xiaodong, Yang Ning, Li Nan, "Trends in small cell enhancements in LTE advanced", Communications Magazine, IEEE, vol.51, no.2, pp.98,105, February 2013

[3] D. Xenakis, N. Passas, L.Merakos, C. Verikoukis, ”Mobility Management for Femtocells in LTE-Advanced: Key Aspects and Survey of Handover Decision Algorithms", Communications Surveys \& Tutorials, IEEE , vol.16, no.1, pp.64,91, First Quarter 2014

[4] K.I Pedersen, P.H. Michaelsen, C. Rosa, Barbera, ”Mobility enhancements for LTE-advanced multilayer networks with inter-site carrier aggregation”, Communications Magazine, IEEE, vol.51, no.5, pp.64,71, May 2013

[5] 3GPP Technical Report 36.839, Evolved Universal Terrestrial Radio Access (E-UTRA); Mobility enhancements in heterogeneous networks, V11.1.0 Available at www.3gpp.org

[6] Doo-Won Lee, Gye-Tae Gil, and Dong-Hoi Kim. "A cost-based adaptive handover hysteresis scheme to minimize the handover failure rate in 3GPP LTE system". EURASIP J. Wirel. Commun. Netw. 2010, Article 6 (February 2010), 7 pages.

[7] Jung-Min Moon, Dong-Ho Cho, "Novel Handoff Decision Algorithm in Hierarchical Macro/Femto-Cell Networks", Wireless Communications and Networking Conference (WCNC), 2010 IEEE , pp.1,6, 18-21 April 2010

[8] Yejee Lee, Bongjhin Shin, Jaechan Lim, Hong, Daehyoung, ”Effects of time-to-trigger parameter on handover performance in SON-based LTE systems", Communications (APCC), 2010 16th Asia-Pacific Conference on , pp.492,496, Oct. 31 2010-Nov. 32010

[9] S. Barbera, P.H. Michaelsen, M. Saily, K. Pedersen, "Mobility performance of LTE co-channel deployment of macro and pico cells", Wireless Communications and Networking Conference (WCNC), 2012 IEEE, pp.2863,2868, 1-4 April 2012

[10] S. Barbera, P.H. Michaelsen, M. Saily, K. Pedersen, "Improved mobility performance in LTE co-channel hetnets through speed differentiated enhancements", Globecom Workshops (GC Wkshps), 2012 IEEE pp.426,430, 3-7 Dec. 2012

[11] T. Jansen, I. Balan, J. Turk, I. Moerman, T. Kurner,"Handover Parameter Optimization in LTE Self-Organizing Networks", Vehicular Technology Conference Fall (VTC 2010-Fall), 2010 IEEE 72nd, pp.1,5, 6-9 Sept. 2010

[12] D. Lopez-Perez, I. Guvenc, Xiaoli Chu, "Theoretical analysis of handover failure and ping-pong rates for heterogeneous networks", Communications (ICC), 2012 IEEE International Conference on, pp.6774,6779, 10-15 June 2012

[13] K. Vasudeva, M. Simsek, and I. Guvenc, "Analysis of Handover Failures in HetNets with Layer-3 Filtering", in Proc. IEEE Wireless Commun. Networking Conf. (WCNC), Istanbul, Turkey, Apr. 2014.

[14] C.H.M. de Lima, M. Bennis, M. Latva-aho, "Modeling and analysis of handover failure probability in small cell networks", Computer Communications Workshops (INFOCOM WKSHPS), 2014 IEEE Conference on , pp.736,741, April 27 2014-May 22014

[15] H. Claussen, L. T. W. Ho, and L. G. Samuel, "An overview of the femtocell concept", Bell Labs Technical Journal, vol. 13, no. 1, pp. 221-246, 2008

[16] 3GPP Technical Specification 36.331 E-UTRA; Radio Resource Control; Protocol Specification, V12.0.0, Mar. 2014 Available at www.3gpp.org

[17] J. Puttonen, J. Kurjenniemi, O. Alanen, "Radio problem detection assisted rescue handover for LTE" Personal Indoor and Mobile Radio Communications (PIMRC), 2010 IEEE 21st International Symposium on ,pp.1752,1757, 26-30 Sept. 2010

[18] 3GPP Technical Report 36.872, Small cell enhancements for E-UTRA and E-UTRAN - Physical layer aspects, V12.1.0, Dec. 2013 Available at www.3gpp.org

[19] 3GPP Technical Report 36.842, Study on Small Cell enhancements for E-UTRA and E-UTRAN; Higher layer aspects, V12.0.0, Jan. 2014 Available at www.3gpp.org 